\title{
Histochemical changes in muscle of individuals with spinal cord injury following functional electrical stimulated exercise training
}

\author{
PD Chilibeck*,1, J Jeon ${ }^{1,3}$ C Weiss ${ }^{1,3}$, G Bell $^{1}$ and R Burnham ${ }^{2,3}$ \\ ${ }^{1}$ The Faculty of Physical Education and Recreation, University of Alberta, Edmonton, Alberta, Canada; ${ }^{2}$ Faculty of \\ Medicine, University of Alberta, Edmonton, Alberta, Canada and ${ }^{3}$ The Rick Hansen Centre, University of Alberta, \\ Edmonton, Alberta, Canada
}

Study Design: Longitudinal training.

Objectives: To determine the effects of functional electrical stimulated (FES) leg cycle ergometer training on muscle histochemical characteristics in individuals with motor-complete spinal cord injury (SCI).

Setting: University of Alberta, Edmonton, Alberta, Canada.

Methods: Six individuals with motor-complete SCI (age 31-50 years; 3-25 years postinjury) trained using FES leg cycle ergometry for $30 \mathrm{~min}, 3$ days per week for 8 weeks. Biopsies of the vastus lateralis muscle were obtained pre- and post-training and analyzed for fibre composition, fibre size and capillarization.

Results: The majority of muscle fibres were classified as type 2 pre- and post-training. Average fibre area increased $23 \%(P<0.05)$ and capillary number increased $39 \%(P<0.05)$ with training. As a result of these proportional increases, capillarization expressed relative to fibre area was unchanged with training.

Conclusions: FES leg cycle ergometer training results in proportional increases in fibre area and capillary number in individuals with SCI.

Sponsorship: Supported by the Glenrose Rehabilitation Hospital.

Keywords: capillarization; vastus lateralis; paraplegia; cycle ergometer

\section{Introduction}

Following spinal cord injury (SCI), paralysed muscle undergoes marked changes, including a shift in fibre composition to fast twitch fibre type ${ }^{1-3}$ muscle fibre atrophy, ${ }^{1,2,4}$ and a reduction in capillary number. ${ }^{4,5}$ These changes may be associated with functional deficits at the whole body level, including a reduced aerobic capacity, ${ }^{6}$ prolonged exercise gas-exchange kinetics, ${ }^{7}$ and reduced insulin-mediated glucose utilization. ${ }^{5}$

Functional electrical stimulated (FES) cycle ergometer training has been used to improve some of these whole-body parameters, including maximal oxygen uptake, ${ }^{8,9}$ gas-exchange kinetics, ${ }^{8}$ and submaximal exercise endurance time ${ }^{9,10}$ in individuals with SCI. In healthy individuals, differences in these measures of exercise peformance have been attributed to differences in muscle characteristics. For example, individuals with a higher proportion of slow-twitch fibres experience a smaller accumulation of metabolic by-products involved in the fatigue process, ${ }^{11,12}$ presumably allowing greater exercise endurance.

*Correspondence: PD Chilibeck, Ph.D., College of Physical Education, University of Saskatchewan, 221 Cumberland Avenue N., Saskatoon, Saskatchewan, Canada S7N 1M3
Also, muscle capillarization is related to maximal oxygen uptake ${ }^{13,14}$ and recovery from exercise. ${ }^{15,16}$ Finally, increases in muscle mass are related to improvements in maximal oxygen uptake and submaximal exercise performance in individuals with initially low muscle mass (ie the elderly). ${ }^{17,18}$ Given the importance of these muscle characteristics for determining exercise capacity in healthy individuals, it was of interest to assess the effects of exercise training, with FES, on the characteristics of paralysed muscle in individuals with SCI.

The purpose of this study was to assess changes in muscle fibre type composition, muscle fibre area and capillarization, with FES cycle ergometer training in individuals with SCI. We hypothesized that this training would favourably induce changes in these muscle characteristics.

\section{Methods}

\section{Subjects}

Six individuals (five males, one female; aged 31-50 years) with motor-complete spinal cord injury (3-25 years post-injury involving levels $\mathrm{C}_{5}-\mathrm{T}_{8}$ ) volunteered for this study. Informed consent was obtained and the 
project was approved by ethics committees of the University of Alberta and the Glenrose Rehabilitation Hospital. Subjects underwent a medical examination prior to participation. Exclusion criteria included subjects with pacemakers, uncontrolled arrhythmias, angina, congestive heart failure, current deep venous thrombosis or pulmonary emboli, severe automomic dysreflexia response to electrical stimulation, less than $90^{\circ}$ of flexion range of motion of the hips and knees, severe lower extremity spasticity, and current participation in regular exercise.

\section{Exercise training}

Subjects trained using a computer controlled FES-leg cycle ergometer (ERGYS II; Therapeutic Alliance, Fairborn, OH, USA) for an accumulated duration of 30 min cycling, 3 days per week, for 8 weeks. Electrical stimulation was applied through surface electrodes $(4.5 \times 10 \mathrm{~cm})$ to the gluteal, hamstring, and quadriceps muscles in a controlled sequence to allow pedalling. Two active electrodes and one reference electrode were placed on each muscle group. Electrical stimulation (monophasic rectangular pulse trains with pulse width of $450 \mu \mathrm{s} ; 30 \mathrm{~Hz}, 10-130 \mathrm{~mA}$ ) was applied to the muscles to enable pedalling at $50 \mathrm{rpm}$. Training sessions began with a warm-up comprised of $1 \mathrm{~min}$ of technician-assisted pedalling at $45 \mathrm{rpm}$. Cycling was initially unloaded for the first training session and an attempt was made to progressively increase resistance over the 8 week period. Following the warm-up, electrical stimulation was increased until subjects were pedalling unassisted at $50 \mathrm{rpm}$. If subjects could not maintain a pedal cadence of $50 \mathrm{rpm}$ with maximal stimulation, resistance was decreased in order for subjects to pedal at $50 \mathrm{rpm}$. If the pedalling rate fell below $40 \mathrm{rpm}$, assistance was provided to finish the next time interval divisible by $10 \mathrm{~min}$. This technician-assisted pedalling would essentially provide an 'overload' stimulus to the muscle, allowing it to continue beyond the occurrence of fatigue failure at $40 \mathrm{rpm}$. This would allow for an increased progression of training. After each exercise interval, a 2 min cooldown (technician-assisted pedalling at $45 \mathrm{rpm}$ ) and 3 min rest period was provided. Training sessions had a maximum of three exercise intervals to complete 30 min of exercise.

\section{Muscle biopsies}

Skeletal muscle biopsies, adapted for suction, were taken from the vastus lateralis at rest before and after the 8-week training program. The tissue was mounted on cork in O.C.T. embedding compound, placed in isopentane cooled to near freezing in liquid nitrogen, and stored at $-80^{\circ} \mathrm{C}$. Sections were cut at a thickness of $10 \mu \mathrm{m}$ at $-20^{\circ} \mathrm{C}$ using a Tissue-Tek Cryostat (Miles Laboratories) and mounted on glass slides with a coverslip. Muscle sections were simultaneously stained for capillaries and fibre types using the technique of Rosenblatt et al. ${ }^{19}$ as modified for human muscle by Hepple et al. ${ }^{13}$ This technique does not allow for differentiation of type 2 fibre subtypes. Staining for each individual's pre- and post-training muscle biopsy were done within the same assay to decrease inter-assay variability. Sections were magnified and projected on a PSI-COM 232 computer-assisted image analysis system (Perceptive Systems Inc., League City, TX, USA) for counting of capillaries and fibres. The number of muscle fibres in sections averaged $177 \pm 93$ (range 68361).

Muscle capillarization was expressed as capillary-tofibre ratio $(\mathrm{C} / \mathrm{F}$; total number of capillaries in a section divided by the number of fibres), the capillary density (total number of capillaries in a section divided by the total fibre area), the average number of capillaries in contact with each fibre (CC), and the average number of capillaries in contact with each fibre divided by the average fibre area (CC/FA). Capillarization was expressed by these four different measures because each offers different information on capillary supply to fibres. When comparisons of capillarization are made across studies, it has been suggested that the best measure to use is $\mathrm{C} / \mathrm{F}$, since a measure such as capillary density is highly influenced by muscle fibre size, which may be affected by shrinkage during histochemical preparation techniques that vary across laboratories. ${ }^{20}$

Capillary density and $\mathrm{CC} / \mathrm{FA}$ are of greater use for assessing capillary supply of fibre area and thus the ability of capillaries to supply oxygen or substrate to muscle, than are $\mathrm{C} / \mathrm{F}$ and $\mathrm{CC}$. $\mathrm{CC} / \mathrm{FA}$ is thought to be the best measure of capillary supply to fibres, as $\mathrm{C} / \mathrm{F}$ and capillary density are global indices of capillarization and yield little information on the capillary supply of individual fibres. ${ }^{21}$

\section{Statistics}

Changes in work rate, duration, and total work output per exercise session, and changes in muscle fibre composition, fibre area, and capillarization were analyzed by dependent $t$-tests. Significance was pre-set at an $\alpha$ level of $<0.05$. All results are expressed as means \pm SEM.

\section{Results}

Training resulted in an increase in mean work rate during exercise from 0 watts at the beginning of the program to $5.1 \pm 2.4$ watts $(P<0.05)$ following 8 weeks. Prior to training, the duration for which subjects were able to continuously pedal the ergometer without assistance averaged $4.3 \pm 0.7 \mathrm{~min}$. Following 8 weeks of training, this was increased to $21.2 \pm 5.6 \mathrm{~min}$ $(P<0.05)$. Average total work output for each training session is shown in Figure 1. This increased from 0 at the first exercise session to $9.2 \pm 4.4 \mathrm{KJ}$ following 8 weeks $(P<0.05)$. 
Cross-sectional samples of muscle fibres, stained for fibre-type and capillaries are shown in Figure 2, for an individual with SCI, prior to (Figure 2b) and following (Figure 2c) the 8-week training program. These are compared to a sample taken from a non-SCI individual (Figure 2a). Compared to this non-SCI individual, the individual with SCI has smaller fibres,

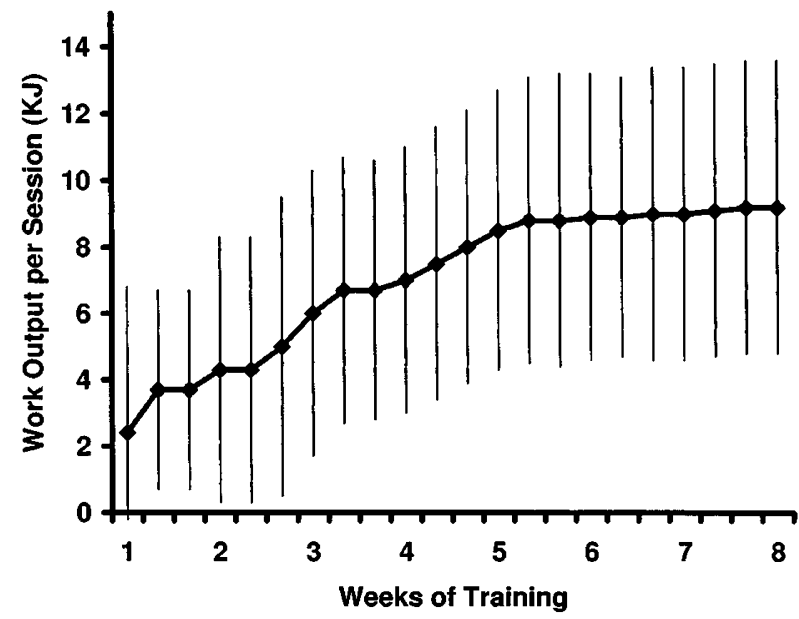

Figure 1 Average work output during each training session over 8 weeks. Error bars are \pm SEM. Work output by week 4 was significantly different from day 1 and a smaller number of capillaries. The non-SCI individual has an equal mixture of type 1 and type 2 fibres, whereas the individual with SCI has a predominance of type 2 fibres. Following the 8-week training program, the most obvious change in the individual with SCI was an increase in fibre size.

For all six subjects with SCI, changes in fibre type composition, fibre size, and measures of capillarization are shown in Table 1. Fibre composition was classified as predominantly type 2 pre- and post-training. Training resulted in a $23 \%$ increase in mean fibre area $(P<0.05)$, a $39 \%$ increase in $\mathrm{C} / \mathrm{F}(P<0.05)$ and a $29 \%$ increase in CC $(P<0.05)$. Since fibre area and capillary numbers increased in proportion to one

Table 1 Changes in muscle fibre characteristics and capillarization (means \pm SEM) with FES-assisted cycle ergometer training

\begin{tabular}{lcc}
\hline & Pre-training & Post-training \\
\hline Capillaries/fibre & $0.75 \pm 0.14$ & $1.04 \pm 0.20^{*}$ \\
Capillary contacts per fibre & $1.30 \pm 0.22$ & $1.68 \pm 0.30^{*}$ \\
Fibre area, $\mu \mathrm{m}^{2}$ & $3428 \pm 729$ & $4206 \pm 756^{*}$ \\
$\mathrm{CC} / \mathrm{FA}, \mu \mathrm{m}^{-2} \cdot 10^{-3}$ & $0.41 \pm 0.05$ & $0.41 \pm 0.05$ \\
Capillaries $/ \mathrm{mm}^{2}$ fibre area & $226 \pm 24$ & $248 \pm 13$ \\
$\%$ type 2 fibres & $91.3 \pm 3.6$ & $88.0 \pm 2.6$ \\
\hline
\end{tabular}

*Post-training value significantly different from pre-training value $(P<0.05)$ a

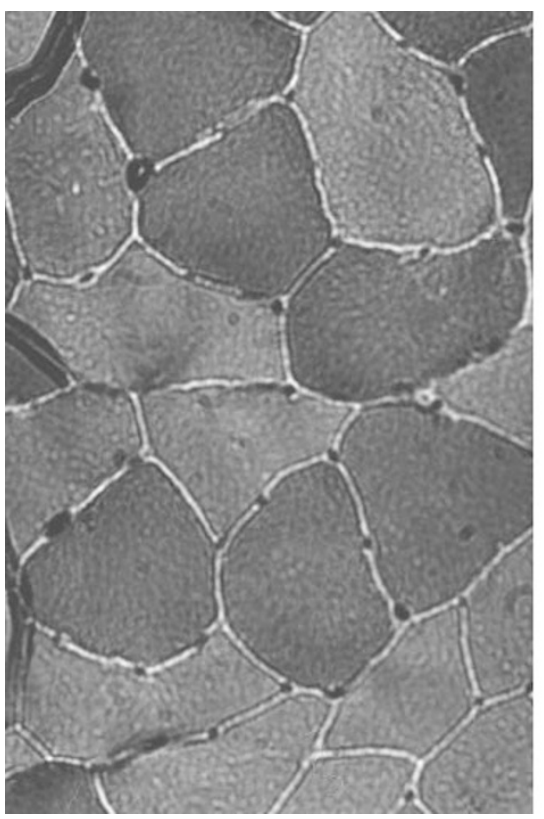

b

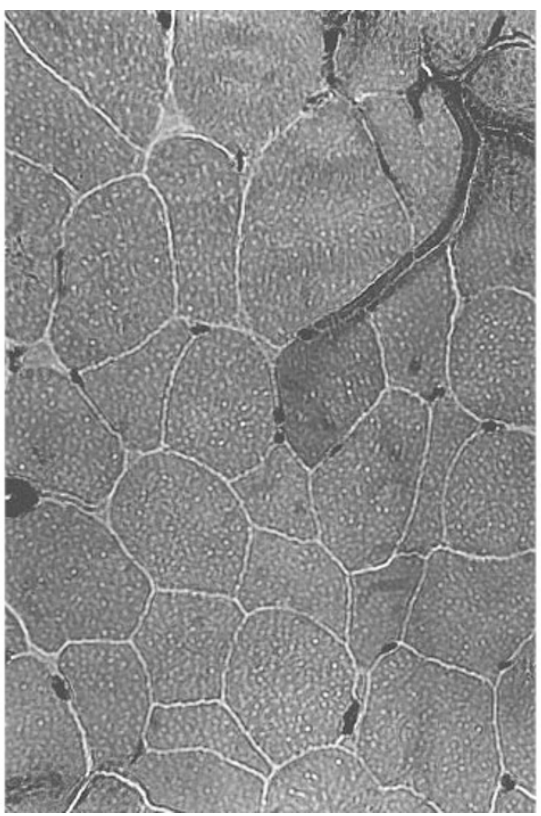

c

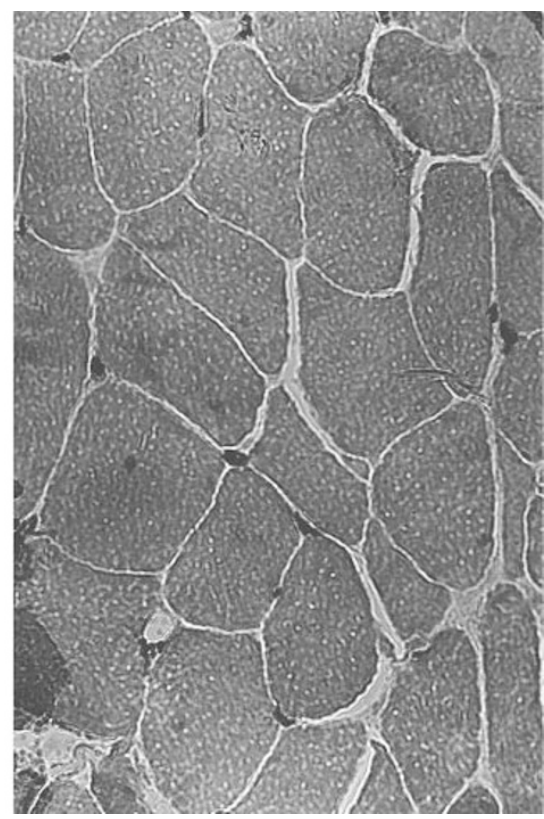

Figure 2 Cross-sections of biopsies of the vastus lateralis stained for fibre type and capillaries for (a) an individual without spinal cord injury (a 22-year-old active male medical school student with fibre type distribution, fibre area, and capillary to fibre ratio which were considered 'average' compared with other healthy young males; ${ }^{15,29,30}$ (b) an individual with spinal cord injury (a 31-year-old male, 8 years since injury at the level of C5) prior to training and (c) the same individual with spinal cord injury following the training program. Type 2 fibres are stained light, and type 1 fibres are stained dark 
another, capillary density and $\mathrm{CC} / \mathrm{FA}$ remained unchanged with training.

\section{Discussion}

To our knowledge, this is the first study to demonstrate that FES-cycle ergometer training increased muscle fibre area and capillary number. Only one other study has assessed changes in muscle fibre characteristics following FES-cycle ergometer training. Mohr et $a l^{9}$ did not find significant changes in muscle fibre area following 6 months of FES-cycling in 10 SCI patients; however, these patients did experience an increase in whole muscle cross-sectional area, as assessed by magnetic resonance imaging. A number of other investigators have also shown that whole muscle cross-sectional area, as measured by computed tomography or magnetic resonance imaging, increases following FES-cycle ergometry or other electrical stimulation protocols. ${ }^{10,22-25}$ As pointed out by Mohr et $a l,{ }^{9}$ without assessment of muscle fibre area, these changes may not reflect a change in muscle fibre mass, but changes in the amount of interstitial tissue. Other stimulation protocols without cycle ergometry have failed to result in changes in muscle fibre area of individuals with SCI. ${ }^{4,26,27}$ Training with electrical stimulation may therefore have to involve work against resistance loads to induce changes in fibre size.

Preventing muscle atrophy in individuals with SCI may be important for increasing whole body glucose clearance, ${ }^{5}$ as skeletal muscle is the major site for glucose disposal in the body. This would be important for preventing the development of type 2 diabetes, which is common in these individuals. ${ }^{28}$ An increase in muscle fibre size should result in a better exercise tolerance in individuals with SCI, as it does for other groups, such as the elderly, that have reduced muscle mass. ${ }^{17,18}$ This was evident in the present study, where power output and duration of exercise increased along with increases in muscle fibre area. An increased exercise tolerance is necessary before workloads, intense enough for increasing cardiovascular fitness, can be accomplished with FES. Improved endurance of paralysed muscle would also contribute to the success of functional tasks such as FES-standing and walking.

Along with an increase in fibre area, our subjects demonstrated a proportional increase in capillary number, with $\mathrm{C} / \mathrm{F}$ increasing by $39 \%$ and $\mathrm{CC}$ increasing by $29 \%$. Previous studies involving electrical stimulation training but without cycling against resistance loads as in the present study, failed to increase muscle capillarization., ${ }^{4,27}$ Training against resistance or simply a longer period of training may be necessary for adaptations to occur. Although our subjects had significant increases in capillary number with training, capillarization was still well below that of healthy non-SCI subjects. The $\mathrm{C} / \mathrm{F}$ of our subjects averaged 1.04 post-training (Table 1 ), while $\mathrm{C} / \mathrm{F}$ for non-SCI individuals ranges from $1.4-2.2 .^{15,29,30}$
Nevertheless, increases in capillarization may be important for adequate delivery of insulin to muscle, ${ }^{31}$ which would increase glucose tolerance, and for the delivery of oxygen ${ }^{15}$ and removal of metabolic by-products such as lactate, ${ }^{11,16}$ which would increase exercise tolerance. The proportional increase in fibre area may, however, offset any benefits of an increase in capillary number, because diffusion distances for oxygen, substrates, or hormones to muscle from capillary would remain unchanged.

Individuals with SCI in the present study had a high proportion of type 2 fibres, with few type 1 fibres, consistent with other studies of SCI. ${ }^{1-3}$ The 8 week program of FES-cycling failed to result in a change in fibre composition. Our results may be limited in that the stain used to simultaneously detect fibre composition and capillaries ${ }^{19}$ does not differentiate type 2 fibre subtypes. There might have been a change in type 2 fibre subtypes but this could not be measured with the methods used. Mohr et al ${ }^{9}$ showed that 12 months of FES-cycling in individuals with SCI results in shifts in myosin heavy chain composition from type $2 \mathrm{~b}$ to $2 \mathrm{a}$, with little change in type 1 myosin heavy chain. Others have shown that 13-24 weeks of daily electrical stimulation, without cycle exercise, induces changes in fibre type from type $2 \mathrm{~b}$ to $2 \mathrm{a}^{26}$ or from type 2 to $1 .^{4}$ These shifts in fibre type composition with electrical stimulation training ${ }^{4}$ are consistent with changes in physiological properties (ie increased indexes of muscle endurance) of muscles in the same subjects. ${ }^{32}$

In summary, 8 weeks of thrice-weekly FES cycle ergometer training results in a proportional increase in muscle fibre area and capillary number. These changes may improve functional parameters, such as muscular endurance in individuals with SCI.

\section{Acknowledgements}

The authors thank Ian MacLean for laboratory assistance. This study was supported by a grant from the Glenrose Rehabilitation Hospital Research Fund.

\section{References}

1 Lotta S et al. Morphometric and neurophysiological analysis of skeletal muscle in paraplegic patients with traumatic cord lesion. Paraplegia 1991; 29: 247-252.

2 Round JM, Barr FMD, Moffat B, Jones DA. Fibre areas and histochemical fibre types in the quadriceps muscle of paraplegic subjects. J Neurol Sci 1993; 116: $207-211$.

3 Burnham $\mathrm{R}$ et al. Skeletal muscle fibre type transformation following spinal cord injury. Spinal Cord 1997; 35: 86-91.

4 Martin TP, Stein RB, Hoeppner PH, Reid DC. Influence of electrical stimulation on the morphological and metabolic properties of paralyzed muscle. J Appl Physiol 1992; 72: $1401-$ 1406.

5 Aksnes AK et al. Intact glucose transport in morphologically altered denervated skeletal muscle from quadriplegic patients. Am J Physiol 1996; 271: E593-E600. 
6 Petrofsky JS, Stacy R. The effect of training on endurance and the cardiovascular responses of individuals with paraplegia during dynamic exercise induced by functional electrical stimulation. Eur J Appl Physiol 1992; 64: 487-492.

7 Barstow TJ et al. Gas exchange kinetics during functional electrical stimulation in subjects with spinal cord injury. Med Sci Sports Exerc 1995; 27: $1284-1291$.

8 Barstow TJ et al. Changes in gas exchange kinetics with training in patients with spinal cord injury. Med Sci Sports Exerc 1996; 28: $1221-1228$.

9 Mohr T et al. Long term adaptation to electrically induced cycle training in severe spinal cord injured individuals. Spinal Cord 1997; 35: $1-16$.

10 Hjeltnes $\mathrm{N}$ et al. Improved body composition after $8 \mathrm{wk}$ of electrically stimulated leg cycling in tetraplegic patients. $\mathrm{Am} \mathrm{J}$ Physiol 1997; 273: R1072-R1079.

11 Tesch PA, Sharp DS, Daniels WL. Influence of fiber type composition and capillary density on onset of blood lactate accumulation. Int J Sports Medicine 1981; 2: 252-255.

12 Mizuno M, Secher NH, Quistorff B. 31P-NMR spectroscopy, rsEMG, and histochemical fiber types of human wrist flexor muscles. J Appl Physiol 1994; 76: 53 - 58.

13 Hepple RT et al. Quantitating the capillary supply and the response to resistance training in older men. Pflügers Arch 1997; 433: $238-244$.

14 Ingjer F. Maximal aerobic power related to the capillary supply of the quadriceps femoris muscle in man. Acta Physiol Scand 1978; 104: $238-240$.

15 Chilibeck PD et al. Muscle capillarization, $\mathrm{O}_{2}$ diffusion distance, and $\mathrm{VO}_{2}$ kinetics in old and young individuals. $J$ Appl Physiol 1997; 82: $63-69$

16 Tesch PA, Wright JE. Recovery from short term intense exercise: its relation to capillary supply and blood lactate concentration. Eur J Appl Physiol 1983; 52: 98 - 103.

17 Fiatarone MA et al. High-intensity strength training in nonagenarians. Effects on skeletal muscle. JAMA 1990; 263: $3029-3034$

18 Frontera WR, Meredith CN, O'Reilly KP, Evans WJ. Strength training and determinants of $\mathrm{VO}_{2}$ max in older men. $J$ Appl Physiol 1990 68: 329-333.
19 Rosenblatt JD et al. A histochemical method for the simultaneous demonstration of capillary and fiber types in skeletal muscle. Stain Technol 1987; 62: 85-92.

20 Plyley MJ, Groom AC. Geometric distribution of capillaries in mammalian striated muscle. Am J Physiol 1975; 228: 1376- 1383.

21 Plyley MJ. Quantifying the capillary supply of skeletal muscle. Can J Sport Sci 1989; 15: 84-85.

22 Pacy PJ et al. Muscle and bone in paraplegic patients, and the effect of functional electrical stimulation. Clin Sci 1988; 75: 481 487.

23 Sloan KE et al. Musculoskeletal effects of an electrical stimulation induced cycling programme in the spinal injured. Paraplegia 1994; 32: 407-415.

24 Block JE et al. Electrically-stimulated muscle hypertrophy in paraplegia; assessment by quantitative CT. J Comput Assist Tomogr 1989; 13: 852 - 854 .

25 Kagaya H, Shimada Y, Sato K, Sata M. Changes in muscle force following therapeutic electrical stimulation in patients with complete paraplegia. Paraplegia 1996; 34: 24-29.

26 Greve JM et al. Functional electrical stimulation (FES): muscle histochemical analysis. Paraplegia 1993; 31: $764-770$.

27 Rochester L et al. Influence of electrical stimulation of the tibialis anterior muscle in paraplegic subjects. 2. morphological and histochemical properties. Paraplegia 1995; 33: 514-522.

28 Duckworth WC et al. Glucose intolerance due to insulin resistance in patients with spinal cord injuries. Diabetes 1980; 29: $906-910$

29 Tesch PA, Thorsson A, Kaiser P. Muscle capillary supply and fiber type characteristics in weight and power lifters. $J$ Appl Physiol 1984; 56: $35-38$.

30 Andersen P, Henriksson J. Capillary supply of the quadriceps femoris muscle of man: adaptive response to exercise. $J$ Physiol 1977; 270: $677-690$.

31 Ader M, Bergman RN. Importance of transcapillary insulin transport to dynamics of insulin action after intravenous glucose. Am J Physiol 1994; 266: E17-E25.

32 Stein RB et al. Optimal stimulation of paralyzed muscle after human spinal cord injury. J Appl Physiol 1992; 72: 1393-1400. 